\title{
SCATTERING CALCULATIONS ON THE BASIS OF THE FREDHOLM INTEGRAL EQUATION METHOD
}

\author{
M. MATSUMURA ${ }^{2}$ AND M. SEK I ${ }^{2}$ \\ 'Astronomical Institute, Tohoku University \\ Sendal 980 , Japan \\ 2 College of General Education, Tohoku University \\ Kawauchl, Sendal 980 , Japan
}

\begin{abstract}
The Fredholm integral equation method (FIM) is one of the solutions to the octtoring of electromagnetic radiation by homogeneous and isotropio ellipsoidal particles. Some numerical calculations are performed with the FIM. The resulta for apherical particles are compared with those by the Mie theory. It is confirmed thet the agreement between them is satisfactory for all the models calculated.

On the basis of the present method, we examine profiles of the absorption band around $\lambda=10 \mu \mathrm{m}$ for apherical and ollipsoidal partioles composed of orystalline olivine. It is found that the profile strongly depends on the shape of the particle. Even when the particle is moderatoly olongated (axial ratios are $2: \sqrt{2}: 1$ ), the profile is oignifioantly different from that for a sphere.
\end{abstract}

\section{Fredholm Integral Equation Method (FIM)}

The dyadicelootric field E of the wave scatered by a particle with a refractive index m can be described by en integration equation

$$
E(r)=I_{1} \exp \left(i k_{\mathbf{I}} \cdot r\right)+\int_{\text {vol }} \mathbf{G}\left(\mathbf{r}, \mathbf{r}^{\prime}\right) \gamma\left(\mathbf{r}^{\prime}\right) \mathbf{E}\left(\mathbf{r}^{\prime}\right) \mathrm{d} \mathbf{r}^{\prime}
$$

where $k_{I}$ is the wave number veotor of the incident light and

$$
\begin{aligned}
& \mathbf{I}_{\mathbf{I}}=\mathbf{J}-\hat{\mathbf{k}}_{1} \hat{\mathbf{k}}_{\mathrm{I}}, \\
& \mathbf{G}=\left(\mathbf{J}+k^{-2} \nabla \nabla\right) \exp \left(i k\left|\mathbf{r}-\mathbf{r}^{\prime}\right|\right) /\left(4 \pi\left|\mathbf{r}-\mathbf{r}^{\prime}\right|\right), \\
& \gamma(\mathbf{r})=k_{0}^{2}\left(m^{2}(\mathbf{r})-1\right),
\end{aligned}
$$

\footnotetext{
-Present addrese: Osaka Museum of Science, Nakanoshima 4-2-1,
} Kitaku, Osaka 630, Japan.

A.C. Levasseur-Regourd and H. Hasegawa (eds.), Origin and Evolution of Interplanetary Dust, 203-206. - 1991 Kluwer Academic Publishers, Printed in Japan. 
where $J$ is a unit dyadic, $\hat{k}_{I}$ is a unit vector along $k_{I}$, and $k$ (ko) is the wave number within (without) the particle. When $r \rightarrow \infty$, the arymptotic form of equation (1) is

$$
E(r)=I_{1} \exp \left(i k_{1} \cdot r\right)+B\left(k_{s}, k_{1}\right) \exp \left(i k_{0} r\right) / r,
$$

where k. is the wave number veotor of the soattered light, and $B$ is the Bottering amplitude given by

$$
B\left(k_{s}, k_{1}\right)=(4 \pi)^{-1} I_{s} \cdot \int_{v o l} \exp \left(-i k_{s} \cdot r\right) \gamma(r) E(r) d r .
$$

Equation (6) Ghow that we can ovaluate numerically B provided that the field within the particle is known. The solution for B within the particle is, however, not obtained directly fromequation (1), since it is an integral equation with a singular kernel. We perform a trangformation suoh that the singularity is removed analytioally. Following Holt et al (1978), we asame that the field within the partiole is expressed as the spatial Fourier transform

$$
E(r)=\int C\left(k_{1}\right) \exp \left(i k_{1} \cdot r\right) d k_{1} \text {. }
$$

Making use of Equation (7), we get a pair of the coupled Fredholm equations, which can be rewritten as matrix equations

and

$$
\mathbf{B}\left(\mathbf{k}_{\mathrm{s}}, \mathbf{k}_{1}\right)=(4 \pi)^{-1} I_{\mathrm{s}} \cdot \sum_{i=1}^{n_{\mathrm{max}}} c_{l} C\left(\mathbf{k}_{l}\right) U\left(\mathbf{k}_{\mathrm{s}}, \mathbf{k}_{l}\right) \text {, }
$$

$$
\sum_{i=1}^{n \text { max }} \mathbf{K}\left(\mathbf{k}_{\jmath}, \mathbf{k}_{\ell}\right) \cdot c_{l} \mathbf{C}\left(\mathbf{k}_{l}\right)=\mathbf{I}_{l} U\left(\mathbf{k}_{\jmath}, \mathbf{k}_{\mathfrak{l}}\right)
$$

where expresitions of $U$ and $K$ are given elsewhere (Holt ot al 1978, Matauma and Seki 1990). Once B is obtained, the oalculations of the optical crose-sections are straightforward as is described in clasical textbooks.

In the practical calculation with the FIM, we must ohoose adequate pivota $k_{1}\left(i=1, \ldots, n_{\max }\right)$. In a spherical coordinate ( $\left.\theta_{p}, \phi_{p}\right)$ whioh displays the directions of $k_{1}$, we first divide evenly the range of $0<\theta<\pi$ by $2 n_{\theta}$, i. e., intervals $\Delta \theta_{P}$ are equal to each other. Next, for each $\theta_{P}$ we divide the range of $0<\phi_{P}<2 \pi$ evenly such that the intervala $\Delta \phi_{P}$ are nearly equal to $\Delta \theta_{F}$. Practically we get $n_{\max }=2$, 12, 30, and 56 for $n_{\theta}=0,1,2$, and 3 (when $n_{\theta}=0$, the pivota are poles only).

\section{Application to silicate $10 \mu$ m band}

We apply the FIM to the investigation of the band profile observed near $\lambda=10 \mu m$ in many astronomioal objects, which is attributed to silicate 
partioles. The shape of the particle is asumed to be spherical or ollipsoidal (the axial ratiog are $2: \sqrt{2}: 1$ ).

Table l presenta values of efficiency factor for absorption Qab as a function of no. The equivalent radius a.a is defined as a.a $=(a b c) 1 / 3$ where a, b, and o are three radil of the ellipaoid, and is at to be 1.0 $\mu \mathrm{m}$ in these oaloulations. We confirm that the convergence for no is rapid, and that the obtained values are correct enough provided thet $\mathbf{n}_{\theta}=1$.

TABLE 1. Examplos of calculations: Qabe for aea=1.0 $2 \mathrm{~m}$

\begin{tabular}{lllll}
\hline$\lambda(\mu \mathrm{m})$ & 9.8 & 10.0 & 11.0 & 11.6 \\
$\operatorname{Re}(\mathrm{m})$ & 0.6109 & 0.6682 & 0.8004 & 2.748 \\
$\operatorname{Im}(\mathrm{m})$ & 0.6344 & 1.061 & 1.379 & 2.291 \\
$\mathrm{X}_{-9}\left(\mathrm{k}_{0} \theta_{-\mathrm{a}}\right)$ & 0.6410 & 0.6283 & 0.6713 & 0.6417 \\
\hline
\end{tabular}

\begin{tabular}{|c|c|c|c|c|}
\hline \multicolumn{5}{|l|}{ Sphere } \\
\hline $\mathbf{n}_{\theta}=0$ & 0.8397 & 1.807 & 2. 366 & 0.8975 \\
\hline 1 & 0.8943 & 1.902 & 2. 415 & 0.8836 \\
\hline 2 & 0.8943 & 1.902 & 2.416 & 0.8836 \\
\hline Mio & 0.8943 & 1.902 & 2. 415 & 0.8835 \\
\hline \multicolumn{5}{|c|}{ Ellipsoid $(2: \sqrt{2}: 1)$} \\
\hline $\mathrm{n}_{\theta}=0$ & 0.8493 & 1.642 & 1.992 & 0.8934 \\
\hline 1 & 0.9347 & 1.793 & 2.100 & 1.119 \\
\hline 2 & 0.9347 & 1.793 & 2.100 & 1.119 \\
\hline
\end{tabular}

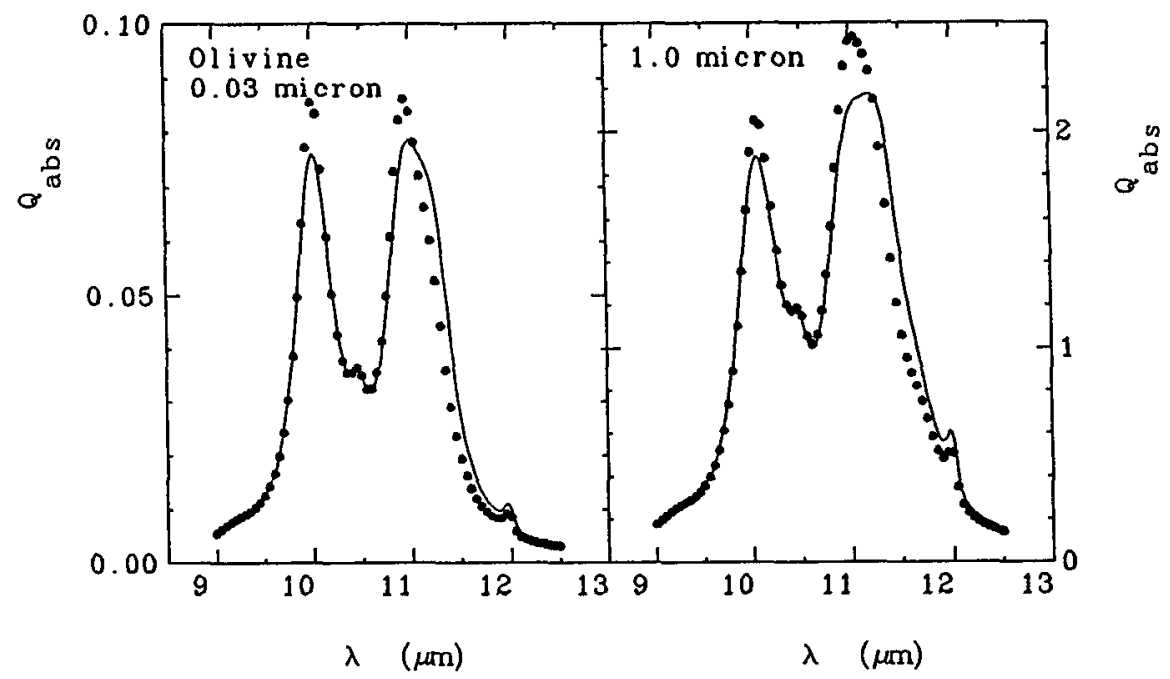

Figure 1. Band profiles for cryotallige olivine. Solid liner are for ellipoidal partioles, while dota arefor spherical ones. The loft figure is for a.a $=0.03 \mu \mathrm{m}$, and the right one ig for a.a $=1.0 \mu \mathrm{m}$. 
Figure I shows the results for crystalline olivine, where optical constanta are oitod from Mukai and Koike (l990). We find that the profile for the ellipsoidal particle (oolid line) is oignificantly different from that for the spherical one (dots).

The resulta for amorphous silioate are demonstrated in figure 2 (m) is from Day 1979). The behavior of the oolid line (resulta for ellipoid) is olosely similar to that of tho dashed line (results for sphere). Thus, the effect of the shape is los oignifioant for amorphous Bilicate.

Recent observations of oomets show a double peak structure in $10 \quad \mu m-$ bend, and this fact is interpreted as the presence of orystalline olivine in cometary grains. The present atudy aggeats that the effect of the shape is important to the quantiative interpretation of such observationa.

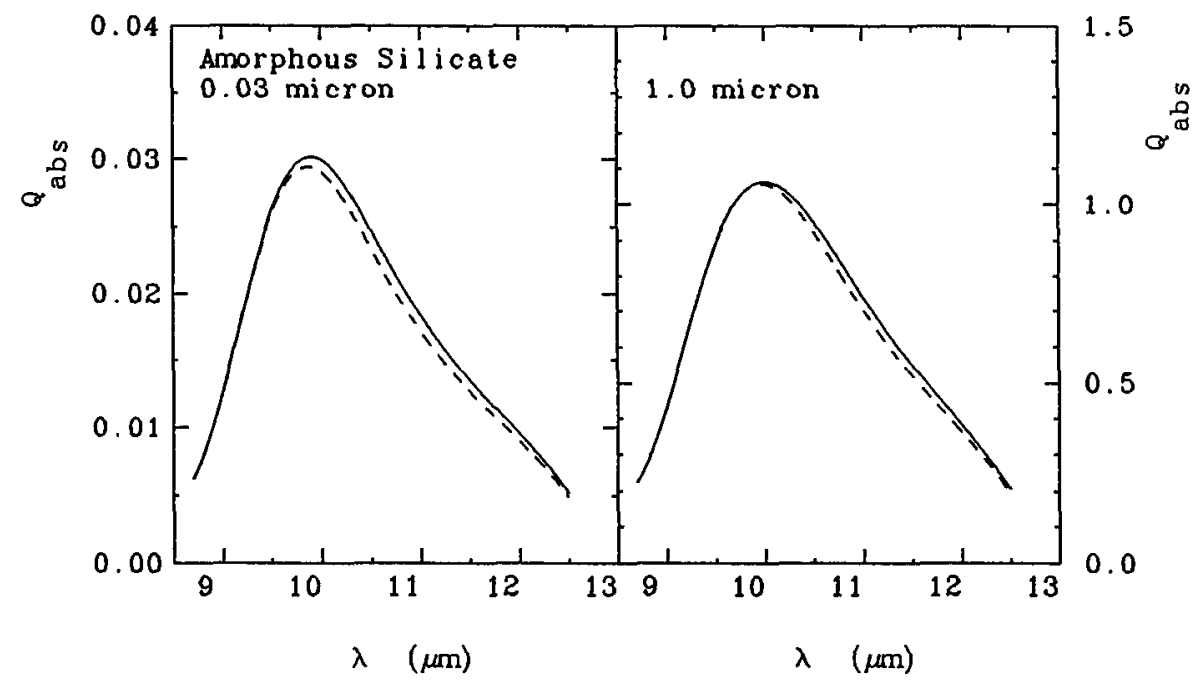

Figure 2. Same as figure 1 but for amorphous ailicate. The reaults for sphere are shown by dashed line.

\section{REFERENCES}

Day, K. L. 1979, Ap. J., 234, 158.

Holt, A. R. , Uzunoglu, N. K. , Evans, B. G. 1978, IEEE Transeotion on Antennar and Propagation 26, 706.

Matoumura, M. , Seki, M. 1990, Ap. Space Soi., in preas.

Mukai, T., Koike, C. 1990, Icarus, in pres. 